\title{
A Integração Ensino-serviço no Contexto dos Processos de Mudança na Formação Superior dos Profissionais da Saúde
}

\author{
Service-learning in the Context of the \\ Changes in the Undergraduate Education of \\ Health Professionals
}

\author{
Verônica Santos Albuquerque \\ Andréia Patrícia Gomes \\ Carlos Henrique Alves de Rezende ${ }^{I I}$ \\ Marcelo Xavier Sampaio ${ }^{I I I}$ \\ Orlene Veloso Dias ${ }^{I V}$ \\ Regina Maria Lugarinho
}

PALAVRAS-CHAVE

- Ensino;

- Serviços de saúde;

- Profissionais de saúde;

- Cuidado de saúde;

- Educaçao médica.

KEY WORDS

- Teaching;

- Delivery of Health Care;

- Health professionals;

- Healthcare practice;

- Medical education.
Recebido em: 15/10/2007

Aprovado em: 15/12/2007 $356 \frac{\text { REVISTA BRASILEIRA DE EDUCAÇ̄̃o MÉDIC. }}{32(3): 356-362 ; 2008}$
${ }^{I}$ Fundação Educacional Serra dos Órgãos, Rio de Janeiro, Brasil.

${ }^{I}$ Universidade Federal de Uberlândia, Minas Gerais, Brasil.

III Universidade Federal do Estado do Rio de Janeiro, Rio de Janeiro, Brasil.

IV Universidade Estadual de Montes Claros, Minas Gerais, Brasil. 


\section{CONSIDERAÇÕES INICIAIS}

Este estudo nasce da sensibilização dos autores para a discussão de questões que envolvem a integração ensino-serviço no contexto dos processos de mudança na formação dos profissionais de saúde.

Entende-se por integração ensino-serviço o trabalho coletivo, pactuado e integrado de estudantes e professores dos cursos de formação na área da saúde com trabalhadores que compõem as equipes dos serviços de saúde, incluindo-se os gestores, visando à qualidade de atenção à saúde individual e coletiva, à qualidade da formação profissional e ao desenvolvimento/satisfação dos trabalhadores dos serviços.

O conteúdo do texto apresentado teve sua origem na construção coletiva de um dos grupos do Curso de Especialização em Ativação de Processos de Mudança na Formação Superior de Profissionais de Saúde, promovido pelo Ministério da Saúde em 2005/2006. O grupo foi constituído por três médicos, duas enfermeiras e um biólogo, inseridos em processos educacionais, de gestão e de atenção à saúde em instituições públicas e privadas nos municípios do Rio de Janeiro (RJ), Teresópolis (RJ), Uberlândia (MG) e Montes Claros (MG).

O grupo refletiu presencialmente e à distância sobre sua própria prática, reconhecendo suas fronteiras de conhecimento, formulando questões, buscando e analisando criticamente novas informações e elaborando estratégias para enfrentar problemas. A produção do grupo foi abrangente, e o presente artigo aborda os aspectos relacionados às reflexões sobre a integração ensino-serviço, assim como os referenciais utilizados para o embasamento teórico de tais reflexões.

Buscou-se abordar a integração ensino-serviço e sua relação com a formação superior dos profissionais de saúde, com os modelos tecnoassistenciais, com a prática do cuidado em saúde, com o trabalho em equipe e com a educação permanente.

\section{OS ESPAÇOS DE INTEGRAÇÃO ENSINO-SERVIÇO COMO CENÁRIOS PRIVILEGIADOS NA FORMAÇÃO SUPERIOR DOS PROFISSIONAIS DE SAÚDE}

Diversas são as perspectivas de mudanças na formação dos profissionais da saúde, as quais incluem a reflexão e transformação da interface ensino/trabalho, ou seja, das relações entre o ensino e os serviços de saúde. Para Henriques ${ }^{1}$, temse visto movimentos na direção de transformações dos velhos modelos de ensino para formação na saúde, os quais se mostram incapazes de responder adequadamente às necessidades apresentadas pela população. Tais movimentos oscilaram, ao longo das duas últimas décadas, na intensidade e na concentração nas diferentes áreas profissionais ${ }^{1}$.
Neste sentido, a formação e o trabalho dos profissionais de saúde na América Latina vêm sendo decisivamente impactados pela reorganização dos sistemas de saúde, pelas pressões para a reforma da universidade e pelo processo de reforma e descentralização político-administrativa do Estado. As iniciativas comprometidas com a relevância social da universidade e dos processos de formação no campo da saúde têm historicamente procurado articular esses dois contextos, aparentemente desconectados — universidade e serviços —, buscando ligar os espaços de formação aos diferentes cenários da vida real e de produção de cuidados à saúde ${ }^{2}$.

Considerando a experiência acumulada nos últimos quarenta anos, particularmente no campo da educação médica, e reconhecendo a inadequação dos modelos de formação para enfrentar os desafios atuais da atenção à saúde, é formado no início dos anos 1990, por iniciativa da Fundação Kellogg, o Programa UNI (Uma Nova Iniciativa na Educação dos Profissionais de Saúde - União com a Comunidade). Sua proposição central se baseou na relação de parceria entre a universidade, os serviços locais de saúde e a comunidade, como o alicerce sobre o qual devem estar fundados os processos de transformação da educação dos profissionais e dos sistemas de saúde. Até então, esses atores estabeleciam entre si relações bilaterais, expressas pela Integração Docente-Assistencial (universidade-serviços), pela Extensão Universitária (universidade-comunidade) e pela Atenção Primária à Saúde (serviços-comunidade). O UNI propõe, em contraposição, articular esses três atores num sistema mais complexo e orientado para a inovação das práticas de saúde e da formação profissional ${ }^{2}$.

Uma análise do Programa UNI permite observar explicitamente uma de suas virtudes: aquela que assume que o processo é de construção e de desconstrução permanente. Nesse caminho, são abordados os avanços inquestionavelmente alcançados, destacando-se a conformação dos projetos enquanto espaços de construção democrática e a conformação de sujeitos orientados por projetos coletivos que resgatam valores como a solidariedade e a responsabilidade compartilhada. Cabe ressaltar um dos conceitos criticados, o de parceria, apresentada como substantiva, horizontal e igualitária entre os componentes (academia, serviços, comunidade). Não foram consideradas as desigualdades de poderes e saberes entre estes componentes, assim como as contradições delas decorrentes. A realidade do desenvolvimento dos projetos revelou, rapidamente, as disputas ocorridas no interior da academia, dos serviços e da comunidade, como nos espaços de negociação entre eles².

Langaná, em 1986, já apontava, como um dos entraves para a interação ensino-serviço, a metodologia baseada na transmissão de conhecimentos, com maior ênfase no ensino do que na aprendizagem, ou seja, reforçando a idéia de que a 
universidade não tem outro papel e/ou compromisso com a sociedade a não ser o de criar, preservar ou transmitir o saber, deixando de lado a missão de atuar na produção de serviços ${ }^{3}$. Olschowsky ${ }^{4}$ complementa, apontando as políticas e estruturas dos serviços de saúde e de ensino como outro fator dificultador dessa interação, já que, muitas vezes, impossibilitam a participação mais efetiva tanto dos profissionais assistenciais como dos docentes na integração ensino-serviço.

Ganha destaque, nesta contextualização da integração ensino-serviço, a discussão de redes como espaços de conformação de um novo ator social. No Brasil, a participação da Rede Unida no processo de definição das diretrizes curriculares, promovido pelo Ministério da Educação, exemplifica o potencial desta articulação. Cabe ressaltar que o enfoque integrado das mudanças organizacionais que tiveram lugar na academia, nos serviços de saúde e nas entidades comunitárias mostra, com clareza, como pode ser fecunda a análise dos processos de transformação das práticas de saúde, quando assentados na interdisciplinaridade ${ }^{2}$.

Cabe ressaltar, ainda, que algumas políticas públicas, como as formuladas pela Secretaria de Gestão do Trabalho e da Educação em Saúde (SGETS), do Ministério da Saúde, apresentaram recentemente possibilidades que se abrem para um conjunto de debates que têm permitido avançar em reflexões e proposições, e que não raro geram novos desafios para continuarmos nossa luta para configurar, nos serviços e nos cursos da área da saúde, os ideais de sociedade justa, ética e igualitária ${ }^{1}$.

Os espaços onde se dá o diálogo entre o trabalho e a educação assumem lugar privilegiado para a percepção que o estudante vai desenvolvendo acerca do outro no cotidiano do cuidado. São espaços de cidadania, onde profissionais do serviço e docentes, usuários e o próprio estudante vão estabelecendo seus papéis sociais na confluência de seus saberes, modos de ser e de ver o mundo.

Centrando a discussão nas relações entre ensino e trabalho, há de se reconhecer que os espaços de interseção entre serviços e ensino são de grande importância para a formação em saúde e para a consolidação do Sistema Único de Saúde (SUS). Neste sentido, Henriques ${ }^{1}$ aborda as conseqüências das práticas que transcendem os cenários de aprendizagem. $\mathrm{O}$ conhecimento ali construído, a partir da reflexão sobre o vivido em um cenário de aprendizagem, pode se difundir por intermédio dos sujeitos que por ali passam como estudantes. Desse modo, são espaços privilegiados para a transformação e consolidação dos modelos de atenção à saúde, pautados pelos valores do SUS. Mas é neles onde também se explicitam conflitos, dificuldades, estratégias e táticas desencadeadas para a ocupação de espaços na rede de cuidados que vai sendo configurada ${ }^{1}$.
Apesar de todas as possibilidades existentes nos espaços de integração ensino-serviço, é inquietante perceber que a formação em saúde - que utiliza os serviços da rede pública de saúde como campo privilegiado para as atividades práticas que compõem essa formação, seja na modalidade de estágio curricular ou na de aula prática - tem uma inserção nesses serviços que se caracteriza por um relativo distanciamento, um tratamento de certa forma cerimonioso entre os envolvidos, no qual as críticas que tenham ao outro não encontram canais adequados de expressão. Dessa forma, sem o diálogo esperado, limitam-se as possibilidades de um fazer diferenciado, que assuma concepções acerca do cuidado, dos processos e organização do trabalho, da gestão e da escuta do usuário ${ }^{1}$.

Deparamo-nos com muitos conflitos decorrentes de problemas e dificuldades na interseção desses dois mundos. Há queixas que dizem respeito, muitas vezes, ao fato de a universidade estar no serviço sem levar em consideração os trabalhadores que lá estão. Tal crítica se amplia quando entra em cena a percepção de que os objetivos acadêmicos estão definidos a priori e não podem se afastar da estrutura já estabelecida. $\mathrm{Ou}$, ainda, que não há participação do profissional do serviço, a não ser na supervisão do estudante, feita em alguns casos de modo assistemático e solitário, sem uma discussão ou presença mais efetiva do docente. Por outro lado, há críticas à diferença marcante entre a lógica da organização dos serviços, muito centrada na produtividade de seus procedimentos técnico-operativos, e a lógica do trabalho da instituição formadora, muito centrada na produção de seus conhecimentos teóricos e metodológicos dos campos pedagógicos e núcleos específicos ${ }^{1}$.

Não é raro observar docentes mais envolvidos com atividades de pesquisa, colocando em segundo plano a prestação de cuidados, o que os distancia das situações práticas do cotidiano e os torna teóricos ineficientes na rotina dos serviços de saúde. Em contrapartida, os profissionais dos serviços, muitas vezes, se envolvem de forma profunda com as atividades rotineiras do cotidiano de trabalho, deixando de lado a educação permanente e, por conseqüência, tornam-se profissionais pouco atualizados ${ }^{5}$.

Tal desarticulação entre teoria e prática suscita a reflexão crítica de que a prática se torna uma exigência da relação teoria/prática, sem a qual a teoria pode ir virando falácia, e a prática, ativismo ${ }^{6}$. Quando a integração ensino-serviço acontece de forma efetiva, unindo docentes, estudantes e profissionais de saúde com o foco central no usuário, esta dicotomia entre o ensino e a produção dos cuidados em saúde se ameniza.

Sendo assim, é preciso investir na sensibilização dos atores inseridos nos cenários onde se desenvolvem os cuidados e o processo de ensino-aprendizagem. Operacionalmente, a universidade deve se preocupar em identificar necessidades dos serviços e cenários de prática, estabelecendo pactos de 
contribuição docente/discente para tais serviços. Devem estar incluídos nestes pactos: negociação de espaços, horários e tecnologias para adequação das atividades do serviço e das práticas educacionais. Além disso, em contrapartida, é fundamental a participação de profissionais dos serviços e usuários nas discussões educacionais de formação na área da saúde.

É necessário criar mais espaços para a interlocução dos cursos, serviços, gestores e, principalmente, usuários ${ }^{1}$. Os profissionais do serviço devem sentir-se co-responsáveis pela formação dos futuros profissionais, assim como os docentes devem considerar-se parte dos serviços de saúde.

Nessa perspectiva, Cecílio ${ }^{7}$ afirma que a passagem dos estudantes pelos serviços tem uma terminalidade. Para se compreender o que o estudante formará sobre o estabelecimento de vínculo entre usuários e serviços, representado pela relação com o profissional que o atende, seria necessário que o professor estivesse no serviço e se sentisse parte dele a ponto de também se ver representado por tal serviço ${ }^{7}$.

Sem o diálogo permanente não será possível gerar novas formas de interferir no processo de trabalho, na organização da assistência, nem no processo educativo da formação de um novo trabalhador. É preciso colocar no centro do diálogo o usuário, que deveria ser o beneficiário dos dois processos que se engendram nos mesmos espaços ${ }^{1}$.

\section{A DIALÉTICA DE FORMAR PARA UM MODELO CENTRADO NO USUÁRIO, INSERINDO ESTUDANTES NA PRÁTICA SOB A ÉGIDE DE UM MODELO MÉDICO HEGEMÔNICO CENTRADO NO PROCEDIMENTO}

Um dos focos da mudança curricular na área da saúde é a formação de profissionais para conformação de um modelo de atenção à saúde centrado no usuário. Porém, a integração ensino-serviço pressupõe a presença de estudantes em formação e docentes em cenários onde ainda se produz atenção à saúde sob um modelo tecnoassistencial hegemônico centrado no procedimento.

Esse modelo predominantemente vigente privilegia a expansão do ensino clínico, especialmente em hospitais, e enfatiza a pesquisa biológica como forma de superar a era empírica do ensino médico, supervalorizando a especialização médica. Este modelo foi criado a partir do relatório Flexner, produzido nos Estados Unidos em 1910 e que mudou o currículo das escolas de medicina, conduzindo a prática médica aos caminhos da superespecialização8.

Associada ao conhecimento especializado, Franco e Merhy ${ }^{9}$ chamam a atenção para a interposição de uma crescente indústria farmacêutica e de equipamentos biomédicos, que eleva consideravelmente os custos com a assistência à saúde.
Podemos definir esse modelo de atenção à saúde como "centrado no procedimento". Isto é, um modelo no qual o principal compromisso do ato de assistir à saúde é com a produção de procedimentos. Apenas secundariamente existe compromisso com as necessidades dos usuários.

O que se busca, atualmente, é fazer o movimento inverso: substituir essas ações custosas de produção da saúde, baseadas em procedimentos especializados e medicalizados, pelas ações relacionais, centradas em atitudes acolhedoras e no vínculo com o usuário, buscando o cuidado à saúde e a cura como finalidade última de um trabalho em saúde que se pauta na defesa da vida individual e coletiva ${ }^{9}$.

A busca deste cuidado integral também mantém relação com o movimento de diversificação de cenários de ensinoaprendizagem, considerada por Feuerwerker ${ }^{10}$ como uma estratégia para induzir mudanças mais profundas no processo de formação profissional; um elemento, em si mesmo, constitutivo de uma nova maneira de pensar esta formação. Não se trata de transformar o espaço dos serviços de saúde e comunidade em prolongamentos dos hospitais-escola, mas, sim, de construir espaços de aprendizagem com a incorporação de docentes e estudantes ao processo de produção de serviços, sem descaracterizar a natureza destes cenários reais.

Neste sentido, a construção de ações e estratégias de controle de doenças e promoção da saúde, de formação de recursos humanos, de controle social, de educação e de comunicação em saúde, de integralidade da atenção, de intersetorialidade e de eqüidade passa a fazer parte das agendas e perspectivas de intervenção de docentes, estudantes e profissionais da saúde ${ }^{10}$.

Neste contexto, cabe ressaltar o papel do trabalhador sobre o modo de fazer "a atenção à saúde": se, de um lado, os fatores sociais, econômicos e políticos definem em grande medida a estrutura e organização dos serviços, a partir de um lugar próprio referente aos aspectos da macropolítica, por outro lado, o funcionamento e o perfil assistencial são dados por processos micropolíticos e pelas configurações tecnológicas do trabalho, mediante os quais ocorre efetivamente a produção do cuidado à saúde ${ }^{9}$.

O trabalho em saúde traz, como componente importante, o fato de que, neste setor, o trabalho humano, vivo em ato, é fundamental e insubstituível. O trabalho ocorre em relações estabelecidas entre os indivíduos trabalhadores e entre estes e os usuários. O "autogoverno" do trabalhador de saúde sobre o modo de fazer assistência, muitas vezes, é o que determina o perfil de determinado modelo assistencial, agindo como dispositivo de mudanças, capazes de detonar processos instituintes ante a organização de serviços de saúde ${ }^{11}$. Por este motivo, a mudança de modelos assistenciais requer, em grande medida, a construção de uma nova consciência sanitária e a 
adesão desses trabalhadores ao novo projeto. É preciso obter consenso sobre formas de trabalhar que estejam em sintonia com a nova proposta assistencial, o que não se consegue por normas editadas verticalmente ${ }^{9}$. Sendo assim, não é possível desconectar a proposta de transformação do modelo tecnoassistencial das mudanças na formação dos profissionais de saúde, com especial destaque, neste sentido, para a integração ensino-serviço como espaço privilegiado de reflexão sobre o ensino e a produção de cuidados.

\section{A INTEGRAÇÃO ENSINO-SERVIÇO COMO ESPAÇO DE REFLEXÃO SOBRE A PRÁTICA DO CUIDADO}

A realidade vivenciada pelos estudantes nos serviços de saúde não deve ser ponto de partida para críticas taxativas a estes serviços e seus profissionais; deve funcionar como elemento instigador para uma prática problematizadora no sentido da aprendizagem e também da reflexão sobre a produção dos cuidados.

É importante que a condução de qualquer análise sobre a relação entre ensino e serviço não busque culpados. Afinal, o que vemos acontecer hoje nos cenários onde se desenvolvem as práticas de formação é fruto de anos de construção que foi traçada, conduzida e consolidada em direções diferentes, com interesses diversos, embora nem sempre antagônicos ${ }^{1}$.

O currículo integrado valoriza o espaço de articulação entre ensino, serviço e comunidade como cenário do processo ensino-aprendizagem, devendo o estudante refletir sobre sua ação e a realidade em que está inserido, buscando problematizar o seu cotidiano, tomando o que tem para ser aprendido como mola propulsora do processo de formação, na perspectiva de uma aprendizagem crítica e reflexiva ${ }^{12}$.

Se considerarmos que os cenários de aprendizagem são de potencial importância como locus da formação em saúde, espaços privilegiados para a incorporação da integralidade no processo de ensino-aprendizagem, a redefinição das práticas e estágios e do seu local de desenvolvimento ganha importância e precisa estar identificada com os princípios políticos e pedagógicos definidos pelos gestores e demais atores do processo educativo. Mas também precisam ser olhados pelos gestores e atores do processo de produção do cuidado como espaço concreto em que as mudanças podem acontecer mutuamente, influenciando e trazendo novos sentidos às suas práticas ${ }^{1,13}$.

O momento de imersão do estudante no cotidiano dos serviços pode trazer recursos riquíssimos para o aprendizado do cuidado e da organização dos processos de trabalho e gestão. Devem-se aproveitar as experiências vivenciadas e observadas nos serviços durante as aulas práticas e estágios, como momento pedagógico, para refletir sobre a prática do cuidado que ali é produzida e suas repercussões, inclusive so- bre a maneira como se concebe o cuidado e se essa concepção se afasta ou se aproxima das manifestações presenciadas naquele espaço. É preciso trazer sentidos para a maneira como a assistência se organiza e desenvolve naquele espaço, onde também estamos ${ }^{1}$.

Neste sentido, o Ministério da Saúde chama a atenção para a formação a partir do processo de trabalho. A formação para a área da saúde deveria ter como objetivos a transformação das práticas profissionais e da própria organização do trabalho e estruturar-se a partir da problematização do processo de trabalho e sua capacidade de dar acolhimento e cuidado às várias dimensões e necessidades em saúde das pessoas, dos coletivos e das populações. A melhor síntese para essa designação à educação dos profissionais da saúde é a noção de integralidade, pensada tanto no campo da atenção, quanto no campo da gestão de serviços e sistemas ${ }^{14}$.

Quando discutimos as mudanças que se fazem cada vez mais necessárias no processo de formação na área da saúde, torna-se essencial que também nos esforcemos por introduzir a discussão de forma mais solidária e companheira sobre as necessidades de mudança na organização dos serviços e de como, na articulação essencial entre os dois mundos — da educação e do trabalho - , temos que definir princípios e valores éticos acerca da saúde e da qualidade dos serviços prestados na área. Percebese a necessidade de um olhar mais elaborado para a questão da interseção entre trabalho e educação, em que os conflitos (dispositivos de poder na construção de modelos e na relação docente/ profissional) presentes nessa interação se devem, parcialmente, aos objetivos e intenções que movem os dois processos ${ }^{13}$.

\section{A INTEGRAÇÃO ENSINO-SERVIÇO PAUTADA NO TRABALHO EM EQUIPE}

O processo educacional na formação dos profissionais da saúde deve ter em vista o desenvolvimento tanto de capacidades gerais (identificadas com a grande área da saúde), quanto daquelas que constituem as especificidades de cada profissão. Entretanto, todo processo educacional deveria ser capaz de desenvolver as condições para o trabalho em conjunto dos profissionais da saúde, valorizando a necessária interdisciplinaridade para a composição de uma atenção que se desloque do eixo - recortado e reduzido - corporativo-centrado, para o eixo - plural e complexo - usuário-centrado.

As propostas de formação e de exercício do trabalho em equipe multiprofissional já estão colocadas como realidade em nossa sociedade para a área da saúde, não cabendo legitimidade a qualquer apelo em contrário. Prova disso é a constância da designação do trabalho em equipe em qualquer circunstância propositiva de elevação da qualidade do trabalho e da formação em saúde. A orientação do trabalho em equipe consta tan- 
to das diretrizes para a formação dos profissionais da saúde, quanto das diretrizes para o exercício profissional no SUS ${ }^{15}$.

Segundo Benevides de Barros e Passos ${ }^{16}$, a clínica só pode ser concebida como transdisciplinar e, por isso, deve subverter o eixo de sustentação dos campos epistemológicos e sua estabilização em unidades disciplinares e em "especialismos". Para Ceccim ${ }^{15}$, este movimento de interseção na equipe multiprofissional de saúde teria, nos recursos e instrumentos terapêuticos de cada corpo de conhecimentos e atos de uma profissão, a oportunidade de compor e inventar a intervenção coletiva, constituindo-se cada desempenho ampliado ou modificado em um desempenho protegido pela condição da equipe, tendo como meta projetos terapêuticos responsáveis pela resolubilidade das ações e dos serviços de saúde.

No aspecto da formação em saúde e das ações profissionais do cuidado, nos reportamos o tempo todo às transformações que devem se dar simultaneamente nos dois processos. Mas costumamos falar da perspectiva isolada de cada profissão da área da saúde, quando as mudanças que precisam acontecer dizem respeito a todas as profissões do campo. E, para além do modo como essas profissões produzem um cuidado, é preciso resultar para o usuário algo bom na perspectiva de seu desejo e expectativa, e ser mais completo e solidário nas ações desenvolvidas por toda a equipe ${ }^{1}$.

O ponto de vista apresentado é o de não haver necessidade de que uma ação profissional se sobreponha à outra, mas que, ao possuírem aspectos que são diversos no seu campo específico de saber e de cuidar, são todas igualmente importantes para o usuário, na capacidade de entendê-lo de modo abrangente, na sua singularidade ${ }^{1}$.

Algumas questões, que se apresentam com freqüência, se referem ao modo como os profissionais da área da saúde não vivenciam, ao longo de sua formação, estratégias que articulem suas atividades e saberes com as dos outros profissionais da equipe. Sempre é referido como um dos momentos de dificuldade, quando se iniciam as atividades profissionais, o aprendizado em que se torna necessário compartilhar com outros profissionais os espaços de atuação e os sujeitos daquela atuação. $\mathrm{O}$ aprendizado gera, muitas vezes, situações extremamente conflituosas nas quais acabam prevalecendo as vaidades individuais em detrimento de um cuidado mais qualificado. Diante desse tipo de conflito, torna-se ainda mais significativo colocar o usuário no centro do debate, pois assim é possível evocar os valores éticos que servem de justificativa última para a atuação de todos os profissionais de saúde, criando um terreno comum para o diálogo ${ }^{1}$.

O espaço de interseção entre serviço e formação é rico em possibilidades para a produção de novos saberes e práticas e também para a aquisição de condutas interprofissionais na produção do cuidado ${ }^{1}$.

\section{A INTEGRAÇÃO ENSINO-SERVIÇO ARTICULADA COM O MOVIMENTO DE EDUCAÇÃO PERMANENTE}

Atualmente, não é possível pensar a interface entre ensino e trabalho sem remeter-se à educação permanente. Neste sentido, é preciso contextualizar a integração ensino-serviço neste movimento: profissionais de saúde, docentes e estudantes devem estar inseridos nas estratégias de educação permanente, tendo em vista melhorar a formação e fortalecer o SUS.

Isto porque a educação permanente possibilita, ao mesmo tempo, o desenvolvimento pessoal daqueles que trabalham na área da saúde e o desenvolvimento das instituições. Além disso, ela reforça a relação das ações de formação com a gestão do sistema e dos serviços, com o trabalho da atenção à saúde e com o controle social ${ }^{17}$.

Possibilidade de transformar as práticas profissionais existe, porque perguntas e respostas são construídas a partir da reflexão de trabalhadores e estudantes sobre o trabalho que realizam ou para o qual se preparam. A educação permanente pode ser entendida como aprendizagem-trabalho, ou seja, ela acontece no cotidiano das pessoas e das organizações. Ela é feita a partir dos problemas enfrentados na realidade e leva em consideração os conhecimentos e as experiências que as pessoas já têm ${ }^{17}$.

A proposta da educação permanente parte de um desafio central, coerente com os propósitos da integração ensino-serviço: a formação e o desenvolvimento devem ocorrer de modo descentralizado, ascendente e transdisciplinar, ou seja, em todos os locais, envolvendo vários saberes. O resultado esperado é a democratização dos espaços de trabalho, o desenvolvimento da capacidade de aprender e de ensinar de todos os atores envolvidos, a busca de soluções criativas para os problemas encontrados, o desenvolvimento do trabalho em equipe matricial, a melhoria permanente da qualidade do cuidado à saúde e a humanização do atendimento ${ }^{17}$.

\section{CONSIDERAÇÕES FINAIS}

Não é possível pensar a mudança na formação dos profissionais de saúde sem a discussão sobre a articulação ensino-serviço, considerando-a um espaço privilegiado para uma reflexão sobre a realidade da produção de cuidados e a necessidade de transformação do modelo assistencial vigente em um modelo que considere como objetivo central as necessidades dos usuários.

Ainda são muitas as críticas bidirecionais entre serviço e ensino. Neste sentido, é preciso ampliar os espaços de diálogo 
e sensibilização de todos os atores envolvidos neste contexto para sua co-responsabilização no que diz respeito à formação de novos profissionais e à prestação de cuidados em saúde.

Para que seja possível construir um novo modo de organizar e praticar a atenção à saúde, é preciso um novo perfil de trabalho e de trabalhadores. A formação e a qualificação dos profissionais da saúde devem ser orientadas pelas necessidades da população. Logo, não cabe mais uma relação distanciada e cerimoniosa entre o ensino e o serviço. Muito pelo contrário, é necessária uma articulação estreita, tendo em vista a transformação das práticas profissionais e da própria organização do trabalho.

Esta transformação pressupõe trabalho em equipe, acolhimento dos usuários, produção de vínculo entre eles e as equipes, responsabilização com a saúde individual e coletiva, atendimento das necessidades dos usuários, assim como resolubilidade dos problemas de saúde detectados.

\section{REFERÊNCIAS}

1. Henriques RLM. Interlocução entre ensino e serviço: possibilidades de ressignificação do trabalho em equipe na perspectiva da construção social da demanda. In: Pinheiro R, Mattos RA orgs. Construção social da demanda. Rio de Janeiro: IMS-UERJ/CEPESC/ABRASCO; 2005.

2. Almeida M, Feuerwerker L, Llanos $M$ orgs. A educação dos profissionais de saúde na América Latina: teoria e prática de um movimento de mudança. São Paulo: Hucitec; Buenos Aires: Lugar Editorial; Londrina: Ed. UEL; 1999.

3. Langaná MTC. Integração docente assistencial. Enfoque 1986; 14(1): 12-5.

4. Olschowsky A. Integração docente assistencial: um estudo de caso. Ribeirão Preto, SP; 1995. Mestrado [Dissertação] — Escola de Enfermagem de Ribeirão Preto, Universidade de São Paulo.

5. Padilha MICS. Análise crítica das causas de integração e/ou desintegração docente assistencial na enfermagem. Rev. Gaúch. Enfermagem 1991; 12(1): 33-7.

6. Freire P. Pedagogia da autonomia. 31.ed. São Paulo: Paz e Terra; 2005.

7. Cecílio LC. As necessidades de saúde como conceito estruturante na luta pela integralidade e eqüidade na atenção em saúde. In: Pinheiro R, Mattos RA orgs. Os sentidos da integralidade na atenção e no cuidado à saúde. 4. ed. Rio de Janeiro: IMS-UERJ/CEPESC/ABRASCO; 2006.

8. Silva Jr. AG. Modelos tecnoassistenciais em saúde: o debate no campo da saúde coletiva. São Paulo: Hucitec; 1998.

9. Franco TB, Merhy EE. Programa de Saúde da Família (PSF): Contradições de um programa destinado à mudan- ça do modelo tecnoassistencial In: Merhy EE, Magalhães Júnior HM, Rimoli J, Franco TB, Bueno WS. O trabalho em saúde: olhando e experienciando o SUS no cotidiano. 3.ed. São Paulo: Hucitec; 2006. p. 53-124.

10. Feuerwerker L. Além do discurso da mudança na educação médica: processos e resultados. São Paulo: Hucitec; Londrina: Rede Unida; Rio de Janeiro: Associação Brasileira de Educação Médica; 2002.

11. Merhy EE. Em busca do tempo perdido: a micropolítica do trabalho vivo em saúde. In: Merhy EE, Onocko R orgs. Agir em saúde: um desafio para o público. São Paulo: Hucitec; 1997.

12. Chirelli MQ. O processo de formação do enfermeiro críticoreflexivo na visão dos estudantes do curso de enfermagem da FAMEMA. 2002. Ribeirão Preto; 2002. Doutorado [Tese] — Escola de Enfermagem de Ribeirão Preto, Universidade de São Paulo.

13. Romano RAT, Henriques RLM. Manifestações da integralidade na formação de enfermeiros: os cenários de aprendizagem como espaço concreto da interlocução entre o ensino e o trabalho. In: Anais do IX Seminário Nacional de Enfermagem [CD-ROM]. Brasília: Associação Brasileira de Enfermagem; 2005

14. Brasil. Ministério da Saúde. Secretaria de Gestão do Trabalho e da Educação em Saúde. AprenderSUS: o SUS e as mudanças na graduação. Brasília: Ministério da Saúde; 2004.

15. Ceccim RB. Equipe de saúde: a perspectiva entre-disciplinar na produção dos atos terapêuticos. In: Pinheiro R, Mattos RA orgs. Cuidado: as fronteiras da integralidade. Rio de Janeiro: Hucitec/ABRASCO; 2004.

16. Benevides de Barros RD, Passos E. A construção do plano da clínica e o conceito de transdisciplinaridade. Psicologia: teoria e pesquisa. 2000; 16(1): 71-80.

17. Brasil. Ministério da Saúde. Secretaria de Gestão do Trabalho e da Educação em Saúde. A educação permanente entra na roda. Brasília: Ministério da Saúde; 2005.

\section{CONFLITOS DE INTERESSE}

Declarou não haver

\section{ENDEREÇO PARA CORRESPONDÊNCIA}

Verônica Santos Albuquerque

Av. Alberto Torres, 111 - 5을 Adar

Alto - Teresópolis

CEP 25964-004 / RJ

E-mail: veronicatere@gmail.com 